NASA Technical Memorandum 100752

\title{
Generalized Pulse Equations for Through-Transmission Evaluation of Arbitrary Multilayered Structures
}

E. James Chern and Hatsumi T. C. Nielsen

January 1990 
$-\cdots$ 
NASA Technical Memorandum 100752

\section{Generalized Pulse Equations for Through-Transmission Evaluation of Multilayered Structures}

E. James Chern

Goddard Space Flight Center

Greenbelt, Maryland

Hatsumi T. C. Nielsen

Department of Electrical Engineering

Stanford University

Stanford, California

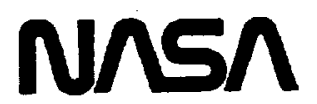

National Aeronautics and

Space Administration

Goddard Space Flight Center

Greenbelt, MD 
Table of Contents

I. Introduction $\ldots \ldots \ldots \ldots \ldots \ldots \ldots \ldots \ldots \ldots \ldots \ldots \ldots \ldots \ldots$

II. Theoretical Derivation and Analysis .......... 2

III. Laboratory Experiments and Results ........... 9

IV. Conclusion $\ldots \ldots \ldots \ldots \ldots \ldots \ldots \ldots \ldots \ldots \ldots \ldots \ldots$

v. References $\ldots \ldots \ldots \ldots \ldots \ldots \ldots \ldots \ldots \ldots \ldots \ldots \ldots \ldots$ 
I 
I. INTRODUCTION

Pulsed ultrasonic techniques are standard methods for detecting and characterizing defects in materials and devices.1-4 To accurately determine the defect parameters and structural integrity of a multilayered structure using ultrasonic techniques, the evaluator must understand the wave propagation in the structure and identify the appropriate signal of interest. Interpreting the signal response is relatively easy with a single-layered specimen, but becomes complicated as number of layers increases, because of multiple pulse reverberations.

We have derived generalized transit time and pulse amplitude formulas for modelling the pulse reverberations in multilayered structures. The equations can be programmed into computer routines and using the appropriate parameters, will calculate the time and echographic amplitude response, which can then be used to identify the critical structural interfaces.

The equations are applicable for both the reflected 5 and the through-transmitted modes. The reflected mode is not applicable in materials and structures that are highly attenuating because the reflected ignals are either buried in the noise or too weak to be detected. As long as the 
material is accessible from the side opposite the transmitter, through-transmission is a viable alternative for evaluating $1088 y$ materials.

In this paper, we show how the generalized formulas for through-transmission time and pulse amplitude response were derived, and present the results of laboratory experiments to verify them. Finally, we discuss the validity of the equations and their applicability to ultrasonic nondestructive evaluation.

Acknowledgement. The first author was previously with Martin Marietta Laboratories. This work was initially supported by the Martin Marietta Laboratories'(MM) Directors Discretionary Fund Program and partially supported by the MII Independent Research and Development Program.

II. THEORETICAI DERIVATION AND ANALYSIS

Stress-wave propagation in multilayered structures is a very complex phenomenon. In addition to complications caused by the normal modes of propagation, other factors such as ourface finish, interface quality, diffraction, scattering, attenuation, focusing, and mode conversion also strongly influence the transmitted and reflected signals. 
Because these factors are mostly material and process dependent, they are very difficult to model mathematically.

In the derivation below, we consider only the pure mode through-transmission of a pulse that is normally incident to a n-layered etructure, which is bounded by two semi-infinite media on each end. Figure 1 illustrates wave propagation in a typical n-layered structure (d is the thickness of the layer and $\alpha$ is the attenuation factor, with the subscript $i$ for the ith layer). The acoustic impedance is given by $z=$ $\rho v$, where $\rho$ is the density of the medium and $v$ is the sound velocity. We assume that all adjacent layers have mismatched acoustic impedances, i.e., $z_{i} \neq z_{i}+1 \cdot$

The reflection and transmission coefficients for an ultrasonic pulse propagated from medium $i$ to medium $f$ are given by $R_{i j}=\left(z_{j}-z_{i}\right) /\left(z_{j}+z_{i}\right)$ and $T_{i j}=\left(2 z_{j}\right) /\left(z_{j}+z_{i}\right)$, respectively. For a single-layered structure, the analysis is otraightforward. The time of arrival for the nth pulse reverberation can be expressed as --

$$
t_{(n)}=(2 n-1) t_{1}=(2 n-1) d_{1} / v_{1}
$$


where $t_{1}=d_{1} / v_{1}$ is the time it takes the pulse to travel through thickness, $d_{1}$, with the wave velocity of $v_{1}$. The pulse amplitude associated with the specific pulse that arrives at $t(n)$ can be written as

$$
A_{(n)}=A_{0} T_{12} R_{10}^{n-1} R_{12}^{n-1} e^{-(2 n-1) d_{1} \alpha_{1}}
$$

where $A_{0}$ is the initial pulse amplitude. As the number of layers in a structure increases, the echo response becomes more complex because the number of reverberations increases and the echo become superimposed. Each transmitted echo $E\left(k_{1}, \ldots, k_{i}, \ldots, k_{n}\right)$ is indexed by the number of times it reverberates in each layer, and apecific echo is represented by a specific set of indices, $k_{i}$. The indices are used to determine the number of echo elements that form the echo. Thus, a pulse response at a given moment may be composed of many echoes that have the same time of arrival. This phenomenon is demonstrated in Figure 2 .

Depending on the phase differences of these echoes, there is either constructive or destructive interference, 
which causes an irregular increase or decrease in amplitude, and which produces local minima and maxima. The formula to compute the number of echo elements is based on the binomial coefficient that determines the number of outcomes that can occur within a given set. A multiplication factor based on pascal's triangle (which can also be derived based on binomial coefficients) specifies the size of a subset of echo elements that follow similar reflection and transmission patterns through the material. The formula is given by --

$$
N=\prod_{j=1}^{n-1} \sum_{i=1}^{\min \left(k_{j} k_{i+1}\right)}\left(\begin{array}{c}
k_{i}-1 \\
j-1
\end{array}\right)\left(\begin{array}{c}
k_{i+1}-1 \\
j-1
\end{array}\right) \text {, where }\left(\begin{array}{l}
m \\
n
\end{array}\right)=\frac{m !}{n !(m-n) !}
$$

Based on the above analysis, we can derive generalized time and amplitude equations for the through-transmission mode. The time response is derived via a linear oummation of the time required to travel through each layer. The 
mathematical expression for the transit time response of a pulse can be written as --

$$
\left(k_{1}, k_{2}, \ldots, k_{n}\right)=\sum_{i=1}^{n}\left(2 k_{i}-1\right) t_{i}
$$

where $k_{1}=1,2, \ldots n$, and $t_{i}=d_{i} / v_{1}$ is the time required for the pulse to travel through the ith layer.

The corresponding amplitude at each calculated time is computed by observing the possible paths the pulse might travel in the given amount of time and is expressed using the $k_{i}$ indices from which the echo elements are determined. Each echo element follows a definite pattern of reflections and transmissions at the material interfaces before it exits to the receiver. By integrating these reflection and transmission coefficients with the results of Equation (3), the echo response at a given time is defined for a given set of indices. The equation for the pulse response amplitude can be expressed as -- 
$A_{\left(k_{1}, k_{2}, \ldots, k_{n}\right)}=$

$$
\begin{aligned}
& A_{0} \frac{T_{n, n+1}}{\prod_{i=2}^{n} T_{i, i-1}} R_{10}^{k_{1}-1} R_{n, n+1}^{k_{n}-1} \theta^{-\sum_{i=1}^{n}\left(2 k_{i}-1\right) d_{i} \alpha_{i}}\left[\prod_{i=1}^{n-1} R_{i, i+1}^{k_{i}+k_{i+1}}\right] \\
& * \prod_{i=1}^{n-1}\left[\sum_{j=1}^{\min \left(k_{i}, k_{i+1}\right)}(-1)^{k_{i+1}^{-j}}\left(\begin{array}{c}
k_{i}-1 \\
j-1
\end{array}\right)\left(\begin{array}{c}
k_{i+1}-1 \\
j-1
\end{array}\right)\left(\frac{1-R_{i, i+1}^{2}}{R_{i, i+1}^{2}}\right)^{j}\right]
\end{aligned}
$$

$A_{0}$ is very difficult to measure experimentally because it depends on several factors, including the transducer characteristics, pulse driver, and coupling efficiency. However, we can normalize the expression for the n-layered structure to $k_{1}=1$ and $k_{n}=1$, i.e., $(n-2)$ layers. Thus, the expression for Equations (4) and (5) reduced to --

$$
t_{\left(1, k_{2}, \ldots, k_{n-1}, 1\right)}=t_{1}+\sum_{i=2}^{n-1}\left(2 k_{i}^{-1)} t_{i}+t_{n}=\left(t_{1}+t_{n}\right)+\sum_{i=2}^{n-1}\left(2 k_{i}^{-1)} t_{i}\right.\right.
$$


and

$$
\begin{aligned}
& A_{\left(1, k_{2}, \ldots, k_{n \cdot 1}, 1\right)}=\left(A_{0} T_{12} T_{n, n+1} e^{-d_{1} \alpha_{1}-d_{n} \alpha_{n}}\right) * \\
& \frac{T_{n-1, n}^{n-1}}{\prod_{i=3} T_{i, i-1}} R_{21}^{k_{2} 1} R_{n-1, n}^{k_{n-1}-1} e^{-\sum_{i=2}^{n-1}\left(2 k_{i}-1\right) d_{i} \alpha_{i}}\left[\prod_{i=2}^{n-2} R_{i, i+1}^{k_{i}+k_{i+1}}\right] * \\
& \prod_{i=2}^{n-2}\left[\sum_{j=1}^{\min \left(k_{i}, k_{i+1}\right)}(-1)^{k_{i+1}-j}\left(\begin{array}{c}
k_{i}-1 \\
j-1
\end{array}\right)\left(\begin{array}{c}
k_{i+1}-1 \\
j-1
\end{array}\right)\left(\frac{1-R_{i, i+1}^{2}}{R_{i, i+1}^{2}}\right)^{j}\right]
\end{aligned}
$$

Equations (6) and (7) are the same expressions as the formulas specifically derived for the $(n-2)$-layered structure. Therefore, the first through-transmitted pulse, which can be measured experimentally can be used as the normalization factor.

The time and amplitude data can be plotted to provide a pictorial presentation of the effect of multiple reverberations that can be used to predict the contribution of significant echoes from the interfaces between layers. Based on the plot, an analysis can be made to determine 
which transmitted pulses are of interest for a specific inspection, and signal processing algorithms can be developed to eliminate unwanted reverberations. 6

III. LABORATORY EXPERIMENTS AND RESULTS

Ultrasonic immersion tests were performed to verify the validity of Equations (4) and (5) for one- and three-layered structures. A benefit of using immersion tests is that it eliminates the error introduced by the thin coupling required in the contact tests. As described in Equations (6) and (7), we first normalized the measurements for a three-layered structure to $t_{1 \mathrm{ml}}$ and $\mathrm{A}_{1 \mathrm{ml}}$ and reduced it to verify the equations for a one-layered structure. Similarly, we normalized the measurements of a five-layered structure to $t_{1 j k 11}$ and $A_{1 j k l}$, and reduced it to three layers.

The equations for the three-layered structure are as follows :

$$
t_{\left(k_{1}, k_{2}, k_{3}\right)}=\left(2 k_{1}-1\right) t_{1}+\left(2 k_{2}-1\right) t_{2}+\left(2 k_{3}-1\right) t_{3}
$$


and

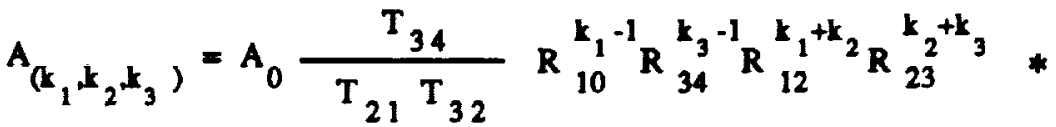

$$
\begin{aligned}
& e^{-\left(2 k_{1}-1\right) d_{1} \alpha_{1}-\left(2 k_{2}-1\right) d_{2} \alpha_{2}-\left(2 k_{3}-1\right) d_{3} \alpha_{3}} \text { * } \\
& {\left[\sum_{i=1}^{\min \left(k_{1}+k_{2}\right)}(-1)^{k_{2}-i}\left(\begin{array}{c}
k_{1}-1 \\
i-1
\end{array}\right)\left(\begin{array}{c}
k_{2}-1 \\
i-1
\end{array}\right)\left(\frac{1-R_{12}^{2}}{R_{12}^{2}}\right)^{i}\right]^{2}} \\
& {\left[\sum_{j=1}^{\min \left(k_{2} \mathbf{k}_{3}\right)}(-1)^{k_{3}-j}\left(\begin{array}{c}
k_{2}-1 \\
j-1
\end{array}\right)\left(\begin{array}{c}
k_{3}-1 \\
j-1
\end{array}\right)\left(\frac{1-R_{23}^{2}}{R_{23}^{2}}\right)^{j}\right]}
\end{aligned}
$$

The reduced expressions for Equations. (8) and (9), i.e., for one layer, are

$$
t_{\left(1, k_{2}, 1\right)}=t_{1}+\left(2 k_{2}-1\right) t_{2}+t_{3}=\left(t_{1}+t_{3}\right)+\left(2 k_{2}-1\right) t_{2}
$$

and 


$$
A_{\left(1, k_{2}, 1\right)}=\left(A_{0} T_{34} T_{12} e^{-d_{1} \alpha_{1}-d_{3} \alpha_{3}}\right) T_{23} R_{21}^{k_{2}-1} R_{23}^{k_{2}-1} e^{-\left(2 k_{2}-1\right) d_{2} \alpha_{2}}
$$

The $t$ ime-domain offset $\left(t_{1}+t_{3}\right)$ and normalization amplitude $A(1,1,1)$ can be measured experimentally.

Three experiments were performed to verify the time and amplitude formulas: two experiments using a three-layered structure (water/0.102-cm glass/water, water/0.131-cm glass/water) and one using a five-layered structure (water $/ 0.318-\mathrm{cm}$ aluminum $/ 0.102-\mathrm{cm}$ water $/ 0.620-\mathrm{cm}$ aluminum/water). The experimental setup is shown in Figure 3. The measurements were taken with a Krautkramer-Branson USIP 12 pulser/receiver and a Tektronix 7904 oscilloscope. Two 10-MHz transducers were used to transmit and receive the ultrasonic pulses. The specimens were immersed in a water bath approximately $1.27 \mathrm{~cm}$ from the transducers.

The acoustic properties of the materials used in these experiments are listed in Table 1. 
Table 1 - Acoustic properties of the materials used in experiments

$\begin{array}{lcc}\text { Material Acoustic Impedance } & \text { Sound Velocity } \\ & \left(\mathrm{kg} / \mathrm{m}^{2} / \mathrm{s}\right) & (\mathrm{m} / \mathrm{s}) \\ \text { Glass } & 14500 & 5710 \\ \text { Aluminum } & 17060 & 6320 \\ \text { Water } & 1483 & 1483\end{array}$

Figures 4 and 5 show the oscilloscope traces of the pulse reverberations for a single-layered $10.102-\mathrm{cm}$ glass plate) sample and a three-layered $(0.318-\mathrm{cm}$ aluminum/0.102cm water/0.620-cm aluminum) composite sample, respectively. The theoretical and laboratory data for these three cases are compared in Figures 6-8. The theoretical calculations of transit time responses predicted using Equation (4) agree well with the experimental measurements. The theoretical calculations of the echo amplitude response [Equation (5)] also agree well with the experimental results for the single-layered samples [Equations (10) and (11), and Figures 6 and 7], but there are discrepancies between the theoretical and experimental results for the multilayered samples. We attribute some of the error 1) to the limited accuracy of the scope, 2) to imperfections in the ourface finishes, 3) to beam spread and dispersion, 4) to imperfect 
alignment of the sensor, and 5) to the superimposing of multiple pulses. Although the experimental measurements are far from ideal, they are adequate to verify the general principles of pulse response as predicted by Equations (4) and (5).

IV. CONCIUSION

It is evident from the results that the generalized equations for through-transmission pulse response in multilayered structures, which are derived here for the first time, are indeed valid. We have verified them using two approaches. First, we reduced the equations to specific forms of single- and three-layered structures and found that the resultant formulas are of the same form as those derived for each cases individually. We also verified the equations experimentally, with generally good agreement between the predicted and experimental measurements of transit time and amplitude response.

These equations are invaluable tools for modeling ultrasonic pulse responses and for practical inspections. They are applicable to both thick and thin specimens, which satisfies the condition $d_{i}>$ wavelength, and can be applied to small electronic components as well as to large 
structures. In addition, they can be easily incorporated into an expert NDE system for evaluating the interfaces of multilayered structures. 
v. RATHRENCES

1. R.C. MaMaster, Nondestructive Testing Handbook (Ronald Pres8, New York, 1959)

2. G.S. Kino, Proc. of the IEEE, 67: 510 (1979)

3. J. Krautkramer and H. Krautkramer, Ultrasonic Testing of Materials, Springer-Verlag, New York (1983)

4. I.M. Brekhovskikh, Waves in Layered Media, Academic Press, New York, (1960)

5. E.J. Chern and H.T.C. Nielsen, J. Appl. Phys. $66(7): 2833,(1989)$

6. D. Kishoni, paper presented at the 1988 Review of Progress in Quantitative Nondestructive Evaluation, La Jolla, California, August (1988) 


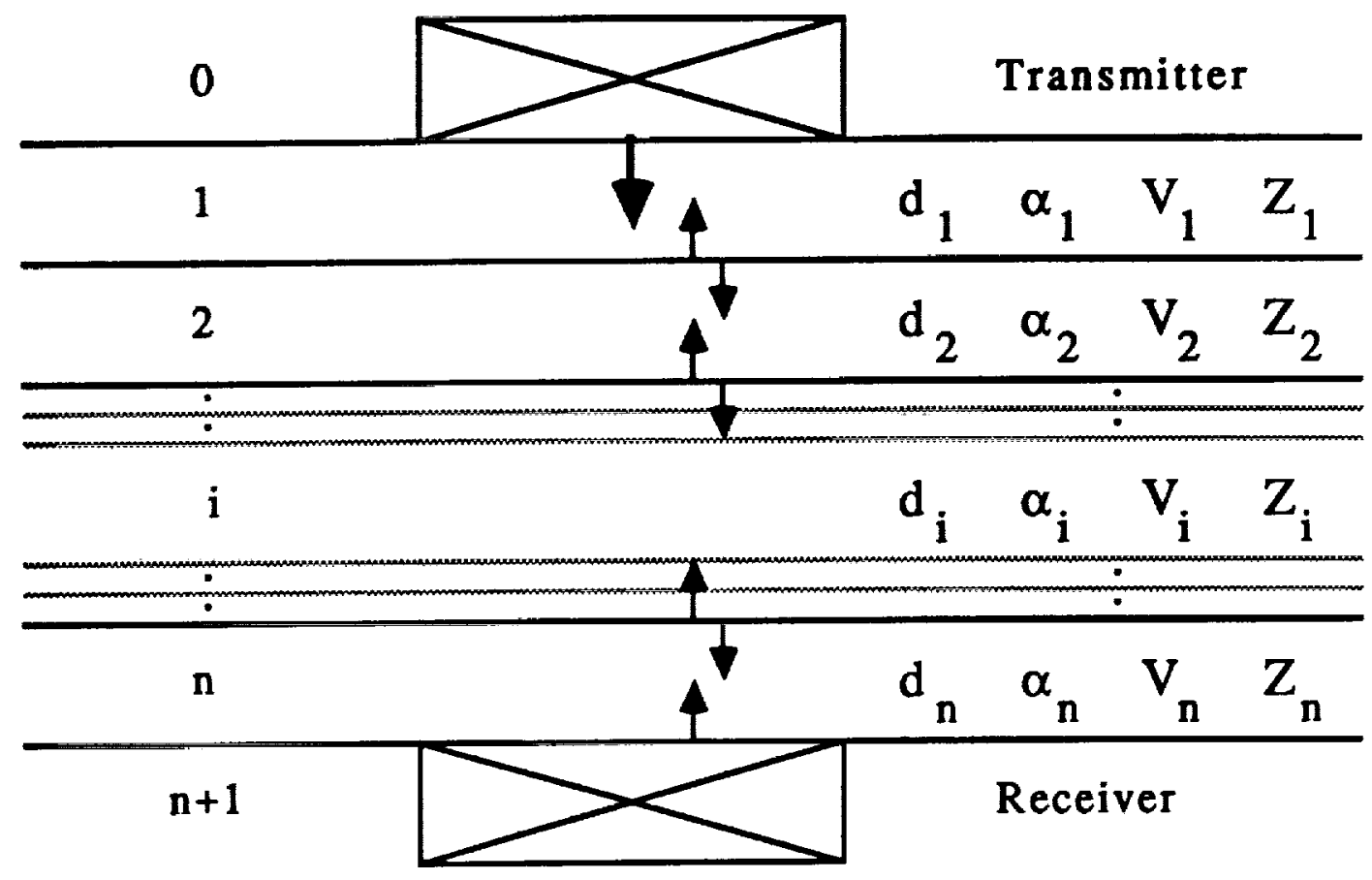

Eigure 1. Wave propagation in a typical n-layered structure. 
For $n=2, \quad k_{1}=3, \quad k_{2}=4$

$N=\left(\begin{array}{l}2 \\ 0\end{array}\right)\left(\begin{array}{l}3 \\ 0\end{array}\right)+\left(\begin{array}{l}2 \\ 1\end{array}\right)\left(\begin{array}{l}3 \\ 1\end{array}\right)+\left(\begin{array}{l}2 \\ 2\end{array}\right)\left(\begin{array}{l}3 \\ 2\end{array}\right)=10$
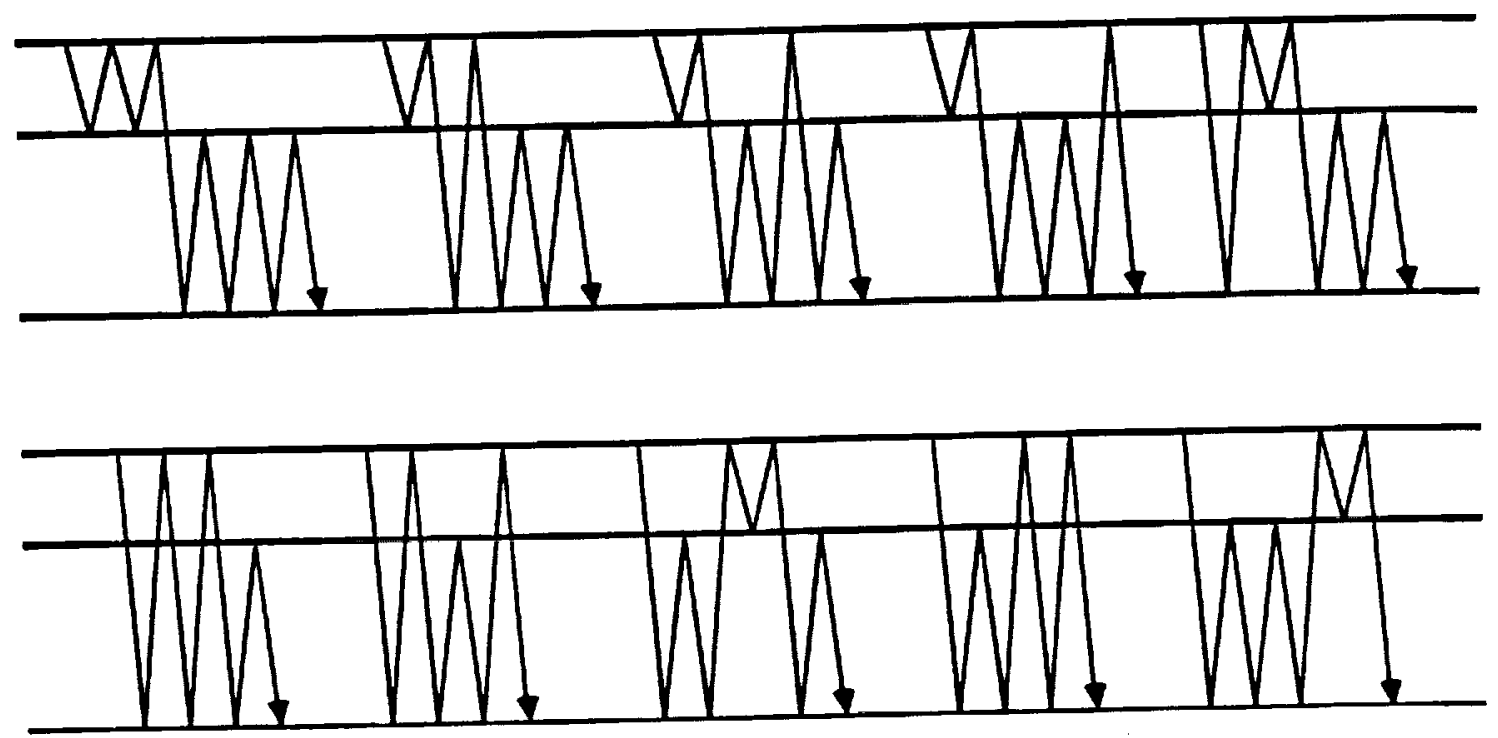

Figure 2. Different wave passages with the same pathlength and arrival time. 


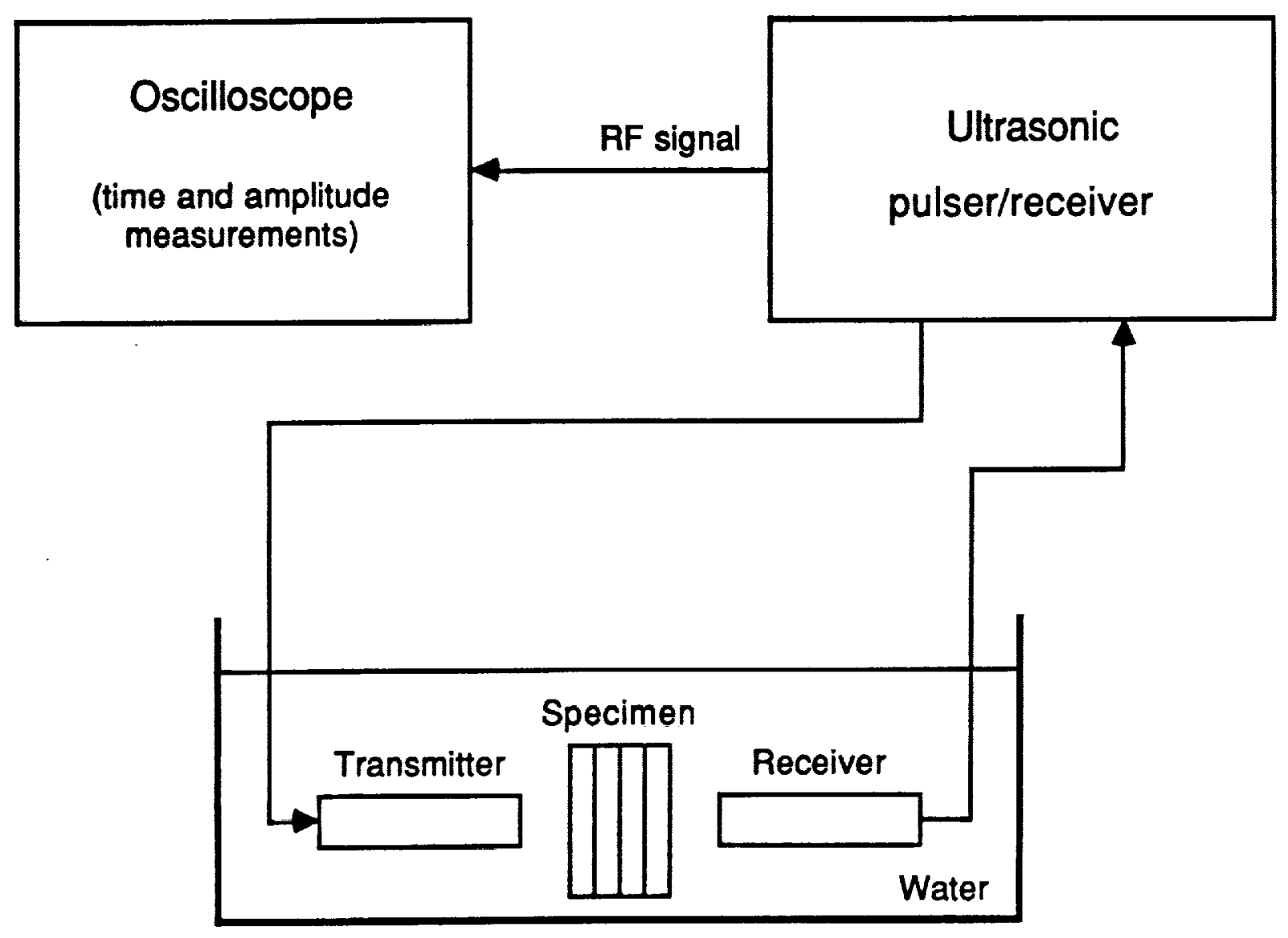

Figure 3. Equipment setup for laboratory experiments. 


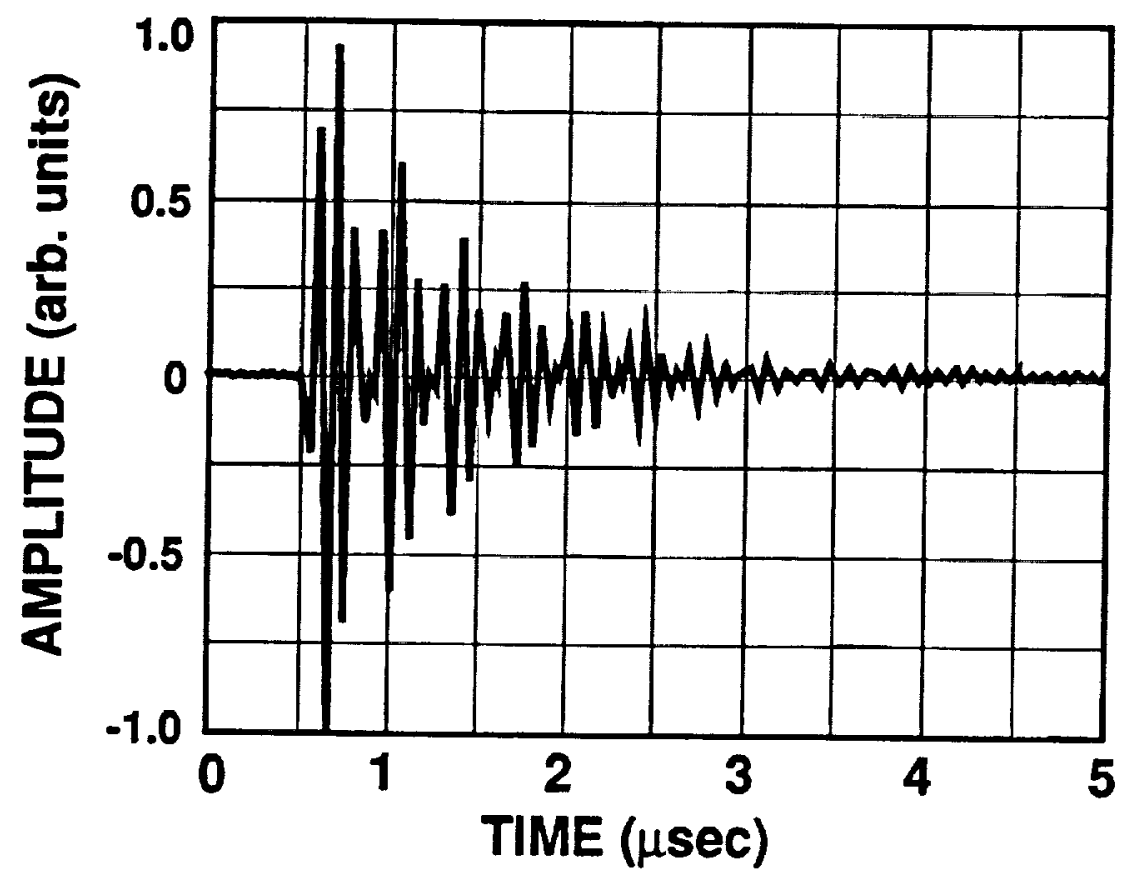

Figure 4. Oscilloscope trace of the pulse reverberation for a single $0.102-\mathrm{cm}-t h i c k$ layer of glass. 


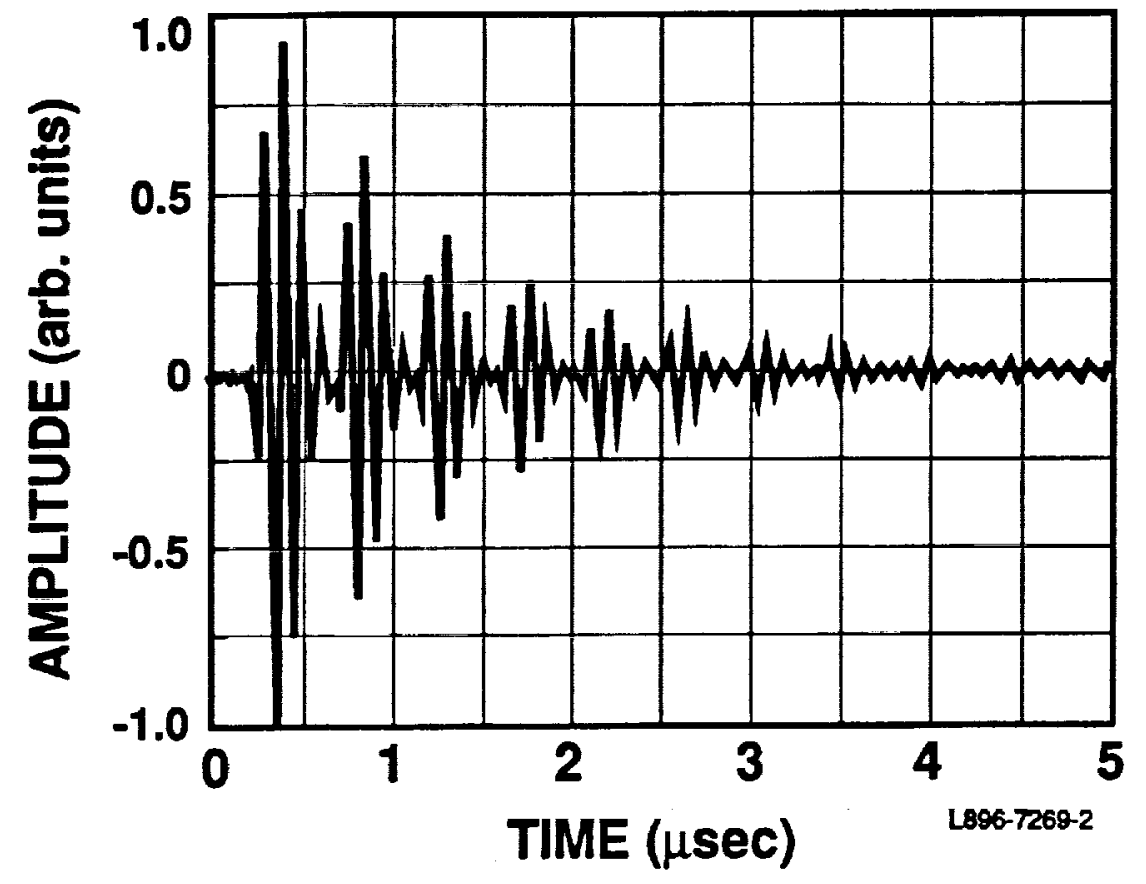

Figure 5. Oscilloscope trace of the pulse reverberation for a three-layered $(0.318-\mathrm{cm}$ aluminum $/ 0.102-\mathrm{cm}$ water $/ 0.620-\mathrm{cm}$ aluminum) sample. 


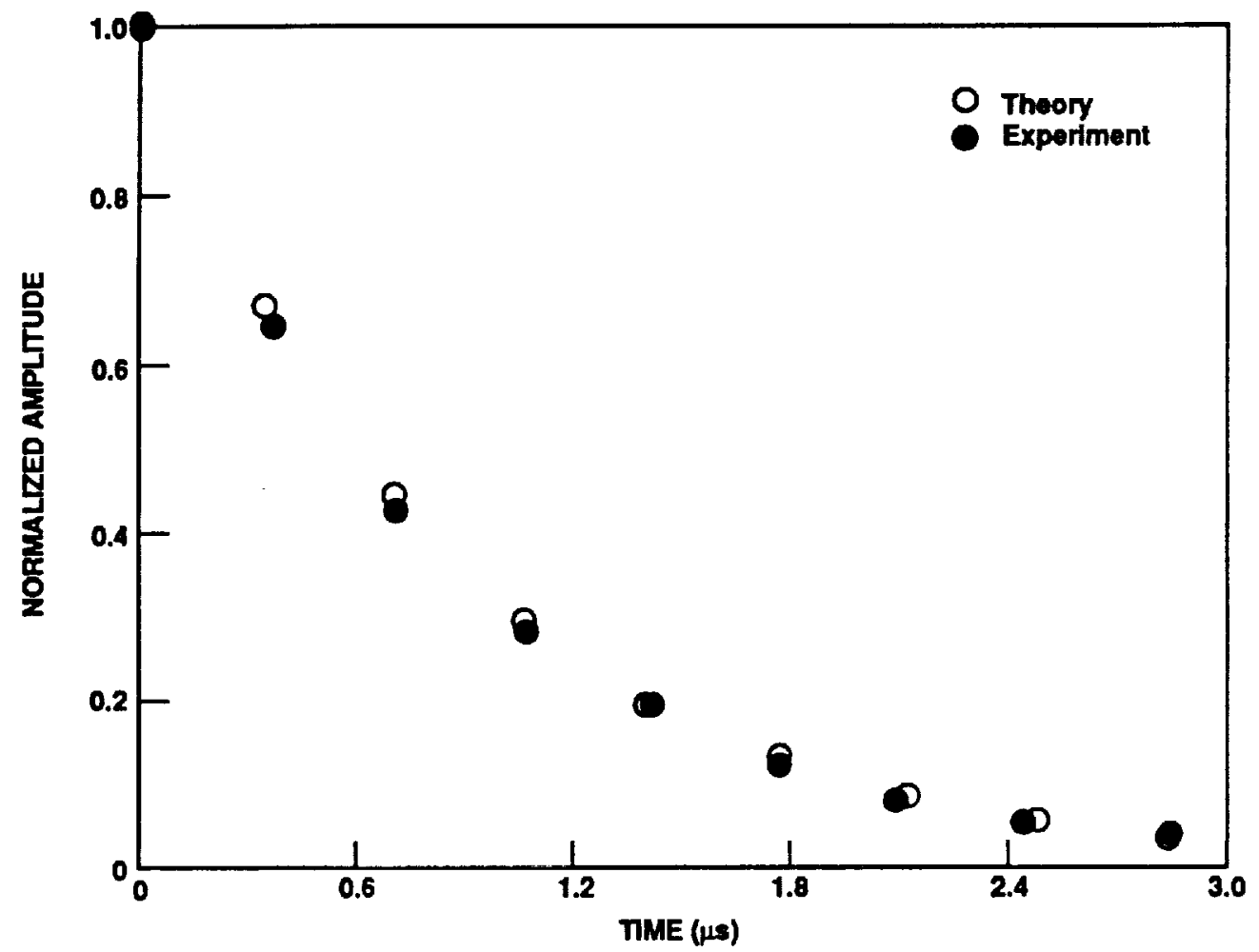

Figure 6. Comparison of the theoretical prediction

[Equations. (10) and (11)] of pulse amplitude and arrival time with experimental results for single 0.102-cm-thick layer of glass. 


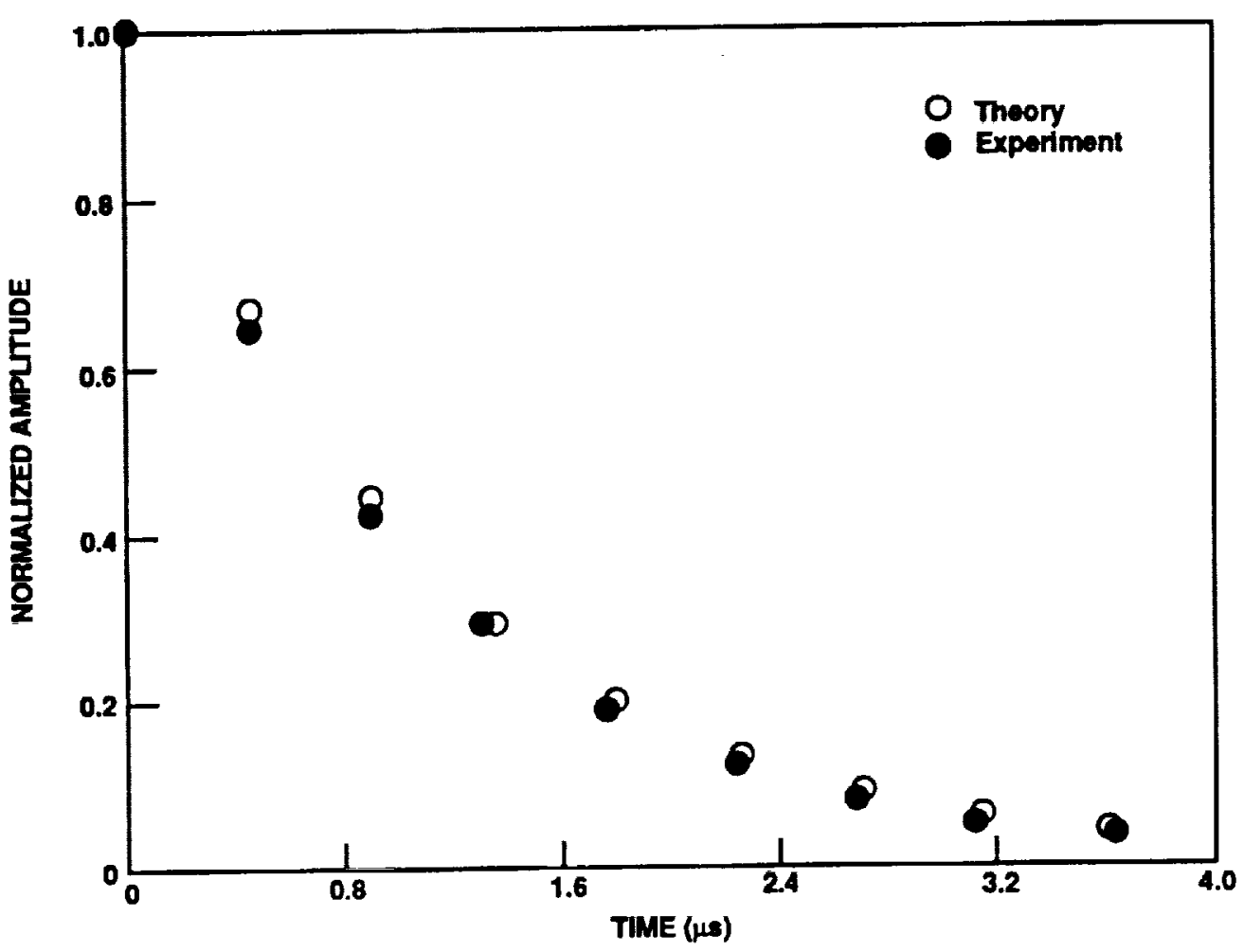

Eigure 7. Comparison of the theoretical prediction of pulse amplitude and arrival time [Equations. (10) and (11)] with experimental results for a single 0.131 -cm-thick layer of glass. 


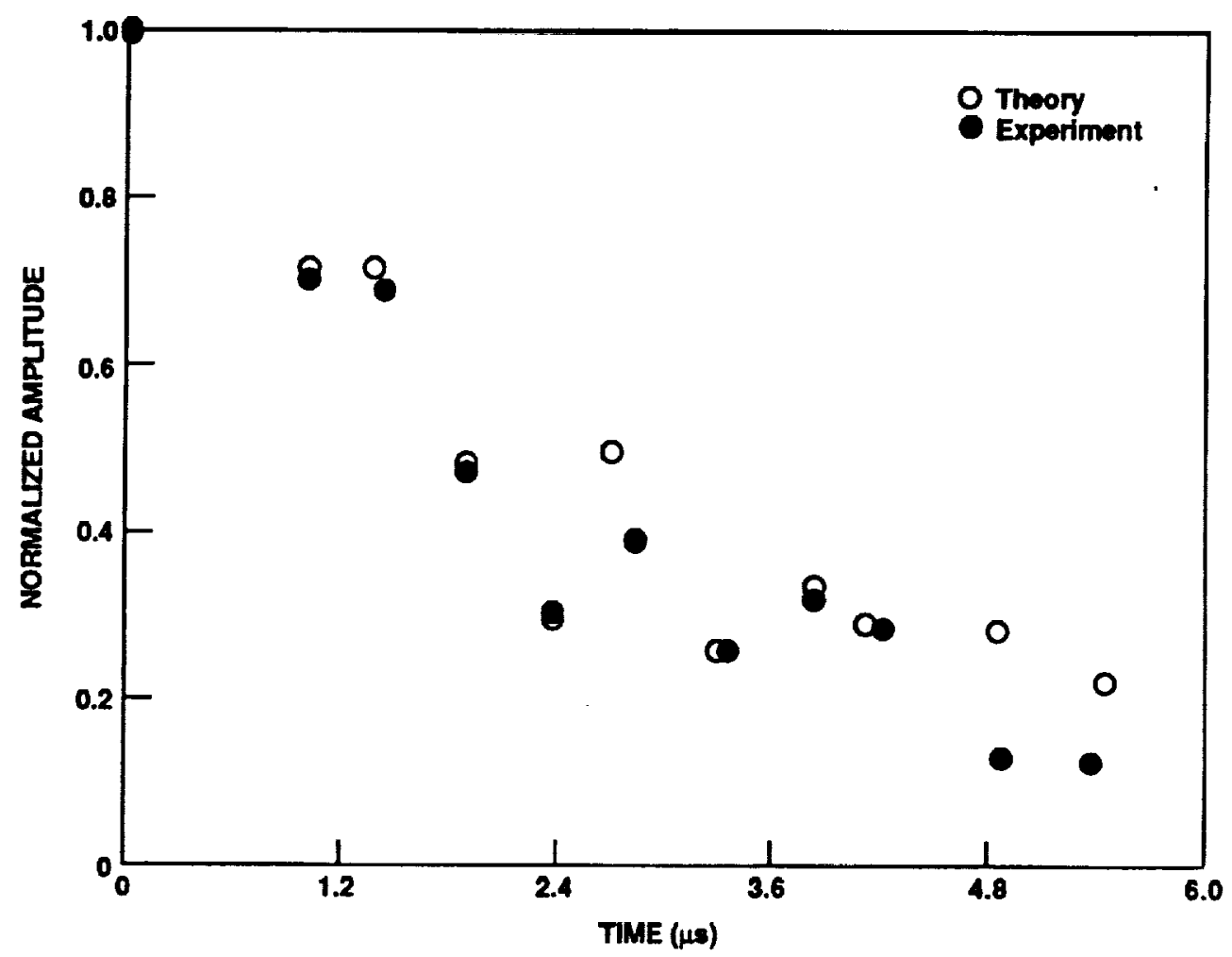

Figure 8. Comparison of the theoretical prediction of pulse amplitude and arrival time [Equations. (4) and (5) ] with experimental results for a threelayered aluminum/water/aluminum sample. 


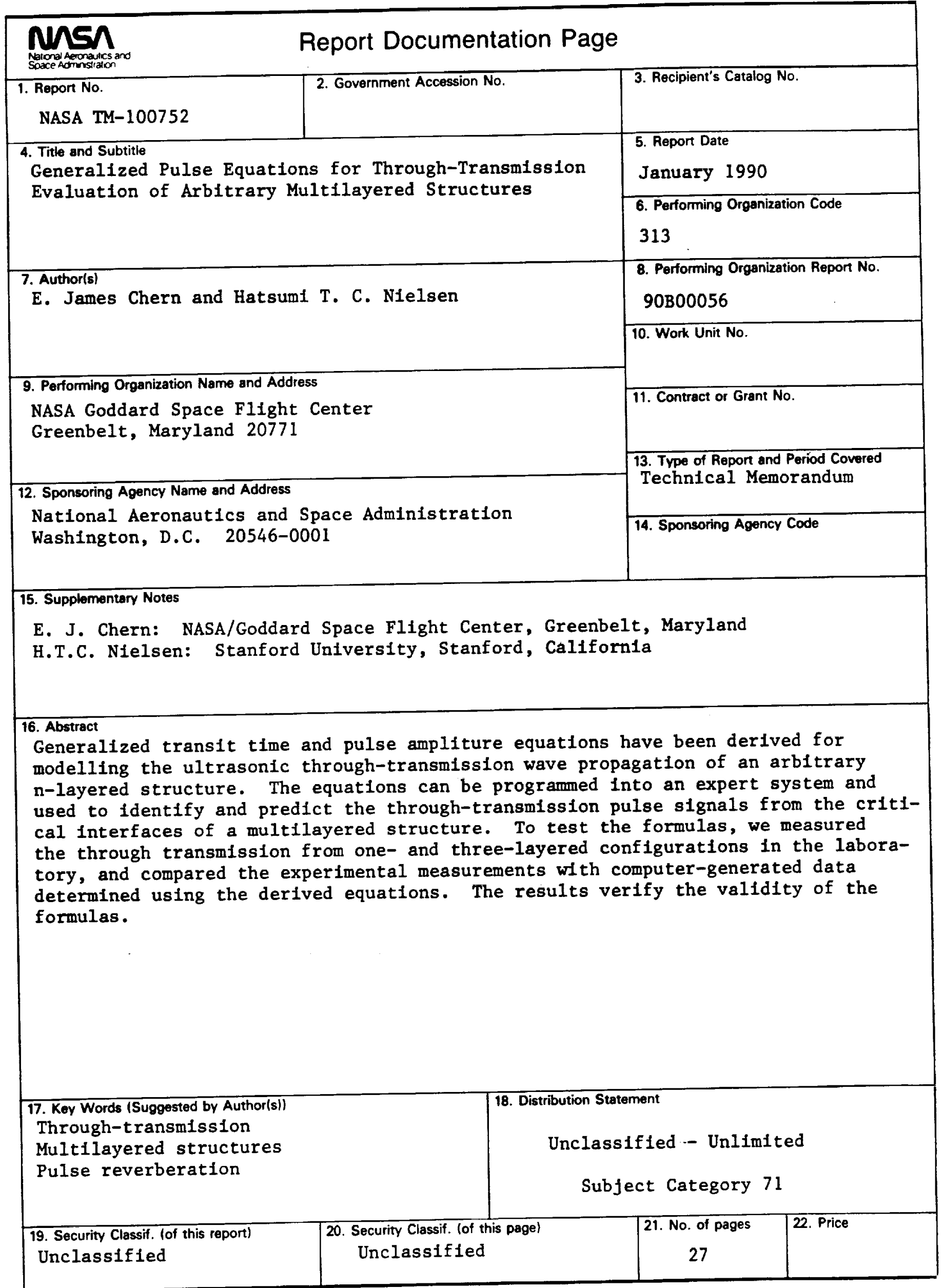




\section{PREPARATION OF THE REPORT DOCUMENTATION PAGE}

The last page of a report facing the third cover is the Report Documentation Page, RDP. Information presented on this page is used in announcing and cataloging reports as well as preparing the cover and title page. Thus it is important that the information be correct. Instructions for filling in each block of the form are as follows:

Block 1. Report No. NASA report series number, if preassigned.

Block 2. Government Accession No. Leave blank.

Block 3. Recipient's Catalog No. Reserved for use by each report recipient.

Block 4. Title and Subtitle. Typed in caps and lower case with dash or period separating subtitle from title.

Block 5. Report Date. Approximate month and year the report will be published.

Block 6. Performing Organization Code. Leave blank.

Block 7. Author(s). Provide full names exactly as they are to appear on the title page. If applicable, the word editor should follow a name.

Block 8. Performing Organization Report No. NASA installation report control number and, if desired, the nonNASA performing organization report control number.

Block 9. Performing Organization Name and Address. Provide affiliation (NASA program office, NASA installation, or contractor name) of authors.

Block 10. Work Unit No. Provide Research and Technology Objectives and Plans (RTOP) number.

Block 11. Contract or Grant No. Provide when applicable.

Block 12. Sponsoring Agency Name and Address. National Aeronautics and Space Administration, Washington, D.C. 20546-0001. If contractor report, add NASA installation or HO program office.

Block 13. Type of Report and Period Covered. NASA formal report series; for Contractor Report also list type (interim, finall and period covered when applicable.

Block 14. Sponsoring Agency Code. Leave blank.

Block 15. Supplementary Notes. Information not included elsewhere: affiliation of authors if additional space is re- quired for block 9, notice of work sponsored by another agency, monitor of contract, information about supplements (film, data tapes, etc.), meeting site and date for presented papers, journal to which an article has been submitted, note of a report made from a thesis, appendix by author other than shown in block 7.

Block 16. Abstract. The abstract should be informative rather than descriptive and should state the objectives of the investigation, the methods employed (e.g., simulation, experiment, or remote sensingl, the results obtained, and the conclusions reached.

Block 17. Key Words. Identifying words or phrases to be used in cataloging the report.

Block 18. Distribution Statement. Indicate whether report is available to public or not. If not to be controlled, use "Unclassified-Unlimited." If controlled availability is required, list the category approved on the Document Availability Authorization Form (see NHB 2200.2, Form FF427). Also specify subject category (see "Table of Contents" in a current issue of STAR), in which report is to be distributed.

Block 19. Security Classification (of this report). Self-explanatory.

Block 20. Security Classification (of this page). Self-explanatory.

Block 21. No. of Pages. Count front matter pages beginning with iii, text pages including internal blank pages, and the RDP, but not the title page or the back of the title page.

Block 22. Price Code. If block 18 shows "UnclassifiedUnlimited," provide the NTIS price code (see "NTIS Price Schedules" in a current issue of STAR) and at the bottom of the form add either "For sale by the National Technical Information Service, Springfield, VA 22161-2171" or "For sale by the Superintendent of Documents, U.S. Government Printing Office, Washington, DC 20402-0001," whichever is appropriate. 